Original Article Journal of Epilepsy Research pISSN 2233-6249 / eISSN 2233-6257

\title{
Determination of Differences in Seed-Based Resting State Functional Magnetic Resonance Imaging Language Networks in Pediatric Patients with Left- and Right-Lateralized Language: A Pilot Study
}

\author{
Audrey Nath, MD, PhD ${ }^{1}$, Meghan Robinson, $\mathrm{MD}^{2}$, John Magnotti, $\mathrm{MD}^{3}$, Patrick Karas, $\mathrm{MD}^{3}$, \\ Daniel Curry, MD ${ }^{4}$, Michael Paldino, MD 5 \\ ${ }^{1}$ Department of Pediatric Neurology, ${ }^{2}$ Core for Advanced MRI, ${ }^{3}$ Department of Neurosurgery, ${ }^{4}$ Division of Pediatric \\ Neurosurgery, Baylor College of Medicine, Houston, TX; ${ }^{5}$ Department of Radiology, University of Pittsburgh \\ Medical Center, Pittsburgh, PA, USA
}

\begin{abstract}
Background and Purpose: The current tools available for localization of expressive language, including functional magnetic resonance imaging (fMRI) and cortical stimulation mapping (CSM), require that the patient remain stationary and follow language commands with precise timing. Many pediatric epilepsy patients, however, have intact language skills but are unable to participate in these tasks due to cognitive impairments or young age. In adult subjects, there is evidence that language laterality can be determined by resting state (RS) fMRI activity, however there are few studies on the use of RS to accurately predict language laterality in children.
\end{abstract}

Methods: A retrospective review of pediatric patients at Texas Children's Hospital was performed to identify patients who have undergone epilepsy surgical planning over 3 years with language localization using traditional methods of Wada testing, CSM, or task-based fMRI with calculated laterality index, as well as a 7-minute RS scan available without excessive motion or noise. We found the correlation between each subject's left and right Broca's region activity and each of 68 cortical regions.

Received December 3, 2019

Results: A group of nine patients with left-lateralized language were found to have greater voxel-wise correlations than a group of six patients with right-lateralized language between a left hemispheric Broca's region seed and the following six cortical regions: left inferior temporal, left lateral orbitofrontal, left pars triangularis, right lateral orbitofrontal, right pars orbitalis and right superior frontal regions.

Conclusions: In a cohort of children with epilepsy, we found that patients with left- and right-hemispheric language lateralization have different RS networks. (2019;9:93-102)

January 24, 2020

Department of Pediatric Neurology, Baylo

College of Medicine, 6701 Fannin St.,

Houston, TX 77030, USA

Tel. $+1-832-822-1750$

Fax. +1-832-825-1717

E-mail; anath@bcm.edu
Key words: Magnetic resonance imaging, functional, Epilepsy, Language

\section{Introduction}

Epilepsy afflicts approximately 470,000 children in the United States. ${ }^{1}$ In the setting of medically-intractable epilepsy, these patients undergo evaluation for the potential surgical resection of epileptogenic foci, which include lesions ranging from focal cortical dysplasias to tubers in tuberous sclerosis complex to regions of perinatal injury. Although potentially curative, one significant barrier to the surgical resection of epileptogenic foci is the presence of eloquent language cortex in the vicinity of the lesion which cannot be resected without causing a permanent language deficit.

Currently, there are several tools available to help localize cortical language function prior to surgery, including cortical stimulation mapping (CSM) using implanted intracranial electrodes, Wada testing, the creation of transient lesions in putative language-based cortex using transcranial magnetic stimulation (TMS), as well as non-invasive methods using magnetoencephalography and task-based functional magnetic resonance imaging (fMRI). In these methods, patients follow commands to perform expressive or receptive language tasks during the creation of transient lesions (CSM, Wada, 
TMS) or during the monitoring of changes in regional electromagnetic activity (as in magnetoencephalography) or blood flow (fMRI).

Despite these advances in language mapping, approximately one quarter of children with epilepsy have cognitive impairments with an intelligence quotient of less than $80,{ }^{2}$ and these cognitive delays often preclude participation in the tasks required for these standard language mapping techniques. These children may have cognitive delays which are intrinsically related to the syndrome causing the epilepsy, such as in the setting of tuberous sclerosis complex, or are unrelated to the cause of epilepsy, such as a co-morbid diagnosis of attention deficit hyperactivity disorder.

Resting state (RS) fMRI is a technique which measures changes in the blood oxygen level-dependent (BOLD) response over 5-10 minutes, in which the subject may be awake and resting or under sedation. ${ }^{3}$ There is a significant literature showing that correlations in the BOLD time course between functionally-coupled areas are higher than between regions without function interactions. ${ }^{4-7}$ It has been shown that language networks may be identified using RS. ${ }^{8}$

While there is evidence in adults that language laterality as determined by RS is comparable to the laterality index (LI) obtained from task-based fMRI (tbfMRI), ${ }^{9,10}$ there have been few previous studies showing that language laterality may be determined in pediatric patients using RS. ${ }^{11}$ There are currently no known previous studies which have investigated differences in RS language networks in children based on language laterality using a seed-based approach. The location of a subject's Broca's region may vary greatly from subject to subject. ${ }^{12,13}$ Therefore, we used a seed-based approach using each individual's cortical anatomy to define Broca's region, then examined differences in each individual's whole-brain connectivity to Broca's region between patients with previouslydetermined left- or right-hemispheric lateralized language.

In this study, we hypothesize that language laterality may be identified due to within-subject differences in connectivity in language networks including left and right Broca's regions.

\section{Methods}

\section{Subjects}

This retrospective study was approved by the Local Institutional Review Board and was compliant with the Health Insurance Portability and Accountability Act. Pediatric patients who had undergone epilepsy surgical planning at Texas Children's Hospital from 2015-2017 were identified for inclusion in this study, which had the following inclusion criteria: 1) pediatric age group, up to 18 years of age, 2) a 3 Tesla MRI examination of the brain, including a resting state fMRI sequence, 3) English-speaking, and 4) language laterality determined unambiguously as left- or right-dominant by Wada, CSM or tbfMRI LI. Subjects were excluded if there were any significant anatomical defects on structural MRI, such as previous hemispherectomy or large regions of encephalomalacia. Of the 203 patients who were admitted to the epilepsy monitoring unit for pre-surgical evaluation during this time period, 39 were given a RS MRI scan, and of these patients, 26 patients had their RS data available for review (the RS data for the other 15 patients was not found), and of these patients, four patients were excluded due to gross anatomical defects from previous cortical resections. Hence, there were 22 remaining patients who met inclusion criteria and were considered for the remainder of the current study procedures.

\section{Structural MRI data acquisition}

Imaging was performed on a $3 \mathrm{~T}$ magnet (Philips, Achieva, Andover, MA, USA) using a 32-channel phased array head coil. For each patient, one structural scan was acquired, consisting of a T1-weighted, three-dimensional volume acquisition fast field echo (repetition time/echo time [TR/TE]: 7.2/2.9 ms; flip angle: 7 degrees; inversion time: $1,100 \mathrm{~ms}$; voxel size: $0.9 \times 0.9 \times 0.9 \mathrm{~mm}^{3}$ ).

\section{Conventional methods for language lateralization}

For each subject, language laterality was determined using separately acquired tbfMRI, Wada testing, CSM, as described below. In one subject, language laterality was determined based on post-operative language ability following surgical resection of left inferior frontal cortex in the vicinity of left Broca's region.

Task-based verb generation fMRI data acquisition and determination of $\mathrm{LI}$

Awake, verb generation tbfMRI for determination of language laterality: testing occurred over several 5-minute sequences using the SensaVue system (Invivo Corporation, Gainesville, FL, USA). All patients underwent a finger-tapping task to establish the reliability of the exam and language tasks. A "\#\#\#" symbol is presented on screen initially, and the patient is instructed to not think of any word or sentence. Then, a noun is presented on screen, and the patient is asked to think of a verb associated with it, without saying this verb or moving his/her lips. Examples are given prior to the presentation of 
the nouns. Scan series contained four blocks of verb generation, separated by 30 seconds of "off" blocks with visual fixation, lasting a total of 4 minutes.

Images were processed using DynaSuite Neuro Software (Invivo Corporation), which involves motion correction, registration of the fMRI data set with T1-weighted MR images, and generalized linear model processing of block paradigm type designs. A LI was computed as follows: (number of left hemispheric voxels - number of right hemispheric voxels) / (sum of left and right hemispheric voxels). Any LI greater than +0.1 was used as a proxy for "left hemispheric" language, and any LI less then -0.1 was used as a proxy for "right hemispheric" language. Patients who had an LI between -0.1 to +0.1 were considered to have "bilateral" language, and as such, were not included in the study.

\section{Wada acquisition}

Please refer to a previous manuscript for the full details of the intracarotid amobarbital procedure in children performed at Texas Children's Hospital. ${ }^{14}$ Briefly, baseline language and memory testing was performed prior to the amobarbital infusion. Amobarbital was then administered into the left and/or right carotid artery, and expressive language (picture naming, sentence repetition and reading) and comprehensive language (ability to execute commands) were tested. A patient was considered to have left- or right-hemispheric dominant language if there was an alteration in language function (such as speech arrest) during amobarbital injection to one hemisphere. If a patient had no speech deficits on either side, then the patient was considered to have "bilateral" language, and hence was not considered for this study.

\section{CSM}

Patients who underwent CSM to localize language cortex had subdural grid electrodes placed for the purposes of both invasive seizure monitoring to aid in seizure localization for surgical planning, as well as to perform language mapping. During language mapping, electrode pairs were stimulated with up to 15 milliamps of current, and interrupted speech at an electrode pair was considered a "positive" result. For the purposes of this study, any patient who had positive language results in the left hemisphere were considered to have left-dominant language, and patients who had positive language results in the right hemisphere were considered to have right-dominant language.

\section{RS analysis}

\section{Data acquisition}

Single-shot echo-planar blood oxygenation level-dependent images were collected (TR/TE: 2,000/30 ms; flip angle: 80; voxel size: $\left.3 \times 3 \times 3.75 \mathrm{~mm}^{3}\right)$. Functional images were acquired in the resting state for 10 minutes (300 volumes) for each patient. Patients were instructed to lie quietly in the scanner with their eyes closed. All images were visually inspected for artifacts, including susceptibility and subject motion.

\section{Data pre-processing}

For each subject, initial data pre-processing was conducted using Analysis of Functional Neurolmages (AFNI) software (National Institutes of Health, Bethesda, MD, USA), ${ }^{15}$ with scripts supplied by afni_proc.py. First, alignment of the functional images to the T1 structural image was performed, with alignment of the third volume of the resting state $\mathrm{fMRI}$ run to the skull-stripped anatomical T1-weighted image. Head motion correction was performed by rigid body registration of each BOLD volume to the third volume. Volumes presenting an estimated motion greater than $0.2 \mathrm{~mm}$ compared to the previous scan were censored from the first level regression. Detrending of the timeseries from each voxel was performed for removal of polynomials up to the 3rd order. De-meaned motion parameters were regressed out of the timeseries from each voxel. Additionally, nuisance signal regression of signal changes from white matter and ventricles was performed. The BOLD images were smoothed with a $5 \mathrm{~mm}$ Full Width at Half Maximum Gaussian smoothing kernel.

As quality control measures, for each subject, the default mode network (DMN) was created using a $5 \mathrm{~mm}$ seed at the midline subparietal sulcus and visually inspected. Subjects with poor DMN distribution by visual inspection were excluded from further analysis. Total signal to noise ratio (TSNR) for the session was also examined to exclude individuals with a TSNR average of less than 200. There was complete overlap between these quality measures.

\section{Within-subject analysis}

For each subject, a cortical surface model was created with FreeSurfer ${ }^{16,17}$ to allow visualization and region-of-interest creation with Surface Mapping with AfNI. ${ }^{18}$ Anatomical region of interest (ROI) to represent the boundaries of left and right Broca's regions were defined as the conjunction of the automatically-parcellated regions of pars opercularis and pars triangularis of the inferior frontal 
lobe of that hemisphere. ${ }^{19}$ Due to known anatomical variability of this region, ${ }^{12,13}$ a manually-chosen $5 \mathrm{~mm}$ radius spherical seed within anatomical boundaries was determined for each subject, based on visual inspection of the connectivity map generated by AFNI's Instacorr feature.

\section{Regional differences}

The maximum correlation values between each Broca's region $\mathrm{ROI}$ seed region and the voxels within each of the 68 automaticallyparcellated regions of cortex in the FreeSurfer Destrieux anatomical atlas were calculated for each subject. Using a correlation coefficient to estimate the functional connectivity between two regions has been described previously in the literature. ${ }^{20,21}$ These 68 maximum correlation values between the left and right Broca's region ROls (136 values total for each subject) were then compared between the left- and right-lateralized language groups using an unpaired $t$-test.

\section{Group analysis}

It is important to note that the comparison of fMRI data from a wide range of ages (from 4-18 years in this study) involves a wide range of both brain sizes ${ }^{22}$ as well as scalp thicknesses. ${ }^{23}$ In order to compare homologous cortical regions across subjects with varying head sizes, subjects were normalized to the Haskins pediatric template $^{24}$ using the auto_tlrc function in AFNI software (National Institutes of Health). Voxel-wise unpaired student's $t$-tests were performed to compare the correlation values between the subjects with pre-determined left-lateralized language versus subjects with pre-determined right-lateralized language.

\section{Results}

\section{Patients}

A total of 22 patients were identified initially based on the inclusion criteria specified for our chart review (see Methods), with 13 having left-lateralized language and nine having right-lateralized language as determined by "gold standard" techniques for determining language lateralization (e.g., Wada testing, CSM, tbfMRI LI). Additionally, one patient in the left-lateralized group had significant motion artifacts, and the RS dataset that was unable to be aligned to the anatomical dataset. Then, a total of three subjects from each language laterality group was removed from further analysis due to excessive noise in the dataset with a TSNR average of less than 200.

In summary, a total of 15 patients (nine patients with pre-

Table 1. Patient demographics, language laterality and location of seizure onset

\begin{tabular}{|c|c|c|c|c|c|}
\hline ID & Group & Method to determine language laterality & Seizure onset location & Gender & Age (years) \\
\hline 1 & Left & CSM & Left frontal & $\mathrm{F}$ & 12.6 \\
\hline 2 & Left & CSM & Left temporal & $\mathrm{F}$ & 14.9 \\
\hline 3 & Left & CSM & Left parietal & $\mathrm{F}$ & 4.7 \\
\hline 4 & Left & CSM & Left frontocentral & M & 12.5 \\
\hline 5 & Left & tbfMRI language LI & Left frontal & $\mathrm{F}$ & 14.8 \\
\hline 6 & Left & tbfMRI language LI & Left temporal & $\mathrm{F}$ & 16.7 \\
\hline 7 & Left & tbfMRI language LI & Left temporal & $\mathrm{F}$ & 18.8 \\
\hline 8 & Left & tbfMRI language LI & Right temporal & $\mathrm{F}$ & 13.6 \\
\hline 9 & Left & Wada & Left frontal & M & 13.0 \\
\hline 10 & Right & CSM & Left centrotemporal & $\mathrm{F}$ & 6.9 \\
\hline 11 & Right & CSM & Left frontal & M & 9.1 \\
\hline 12 & Right & CSM & Left temporal & $\mathrm{F}$ & 8.8 \\
\hline 13 & Right & CSM & Left temporoparietal & M & 11.5 \\
\hline 14 & Right & tbfMRI language LI & Left temporal & $\mathrm{F}$ & 16.4 \\
\hline 15 & Right & Wada & Midline central & M & 14.7 \\
\hline
\end{tabular}

"ID" refers to the subject identifier number, "Group" refers to the subject's assignment to the left or right language laterality groups, as determined by the method in the next column. The seizure onset location for the patient was determined by scalp EEG and/or intracranial EEG recordings.

CSM, cortical stimulation mapping; F, female; M, male; tbfMRI, task-based functional magnetic resonance imaging; LI, laterality index; EEG, electroencephalography. 
determined left-lateralized language and six patients with predetermined right-lateralized language) met inclusion criteria for this study, had complete datasets available for analysis, and had acceptable levels of noise in their RS datasets. The age, gender, seizure onset location (as determined by prior long-term electroencephalography [EEG] monitoring) and predetermined language laterality group are shown in Table 1. The majority of the 15 patients in this study (eight of the nine patients with left-lateralized language and five of the six patients with right-lateralized language) had seizure onset locations within the left hemisphere. There were no significant differences be- tween the left-lateralized and right-lateralized language group subjects in terms of age (average ages 13.5 years and 11.2 years respectively, unpaired $t$-test $p=0.28), \mathrm{RS}$ data signal-to-noise ratio (average TSNR 295 and 252 respectively, unpaired $t$-test $p=0.84$ ), or location of the left and right Broca's region seeds (left Broca average $x$-coordinates 44 and 42 respectively, y-coordinates -39 and -37 respectively, z-coordinates 23 and 19 respectively, and right Broca's region average $x$-coordinates -46 and -50 respectively, $y$-coordinates -31 and -34 respectively, z-coordinates 23 and 19 respectively, all with unpaired $t$-test $p$-values between 0.17 and 0.71 ).

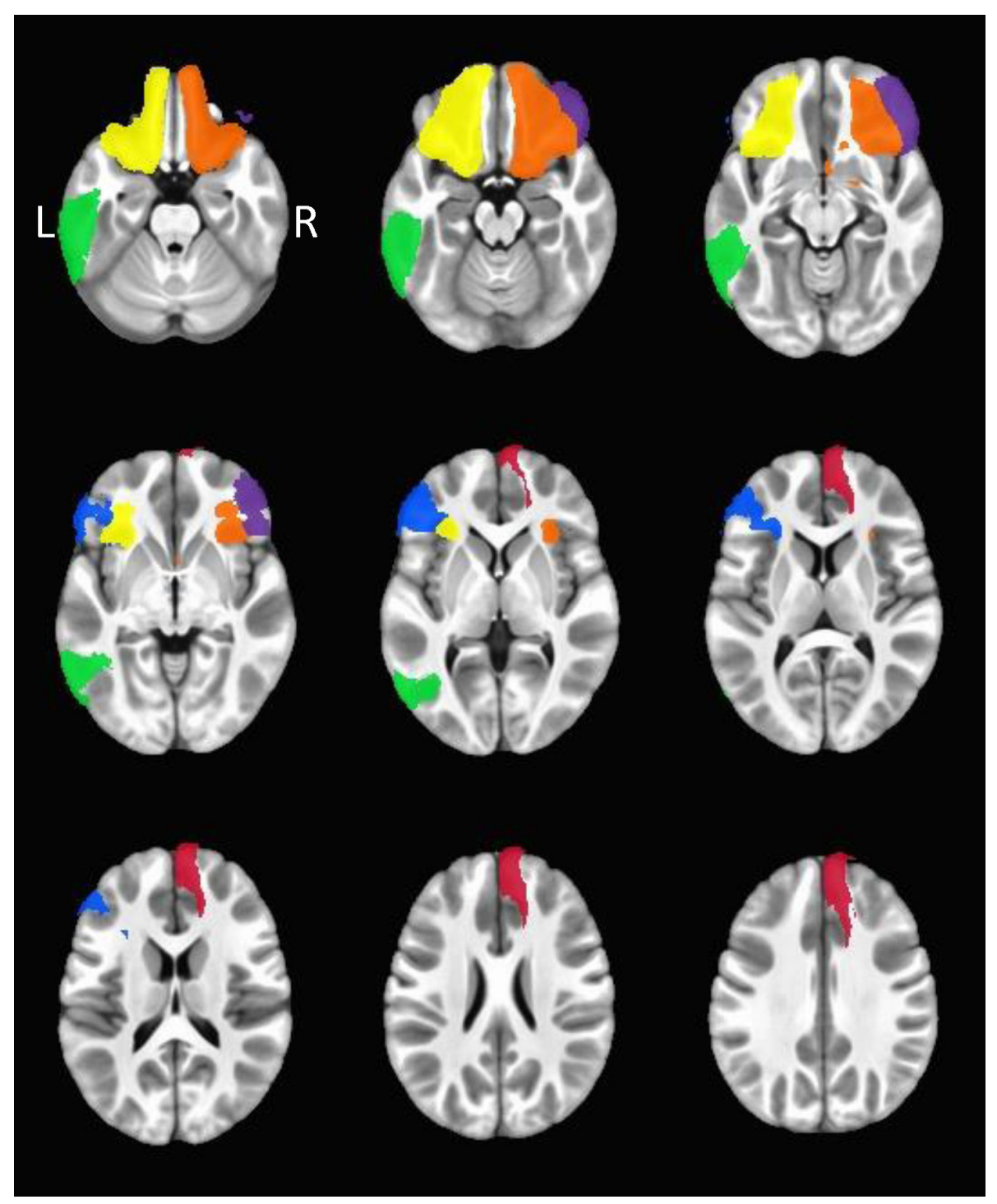

Figure 1. Group analysis, left Broca seed: regions with significantly greater maximum correlations between the left Broca's area seed between in the left-lateralized language group compared to the right-lateralized language group. The regions are colored as follows: left inferior temporal (green), left lateral orbitofrontal (yellow), left pars triangularis (blue), right lateral orbitofrontal (orange), right pars orbitalis (purple), and right superior frontal (red). The left hemisphere of each brain is represented on the left. A montage of axial view slices is shown, with the Haskins pediatric template as the anatomy. 


\section{Resting state fMRI analyses}

Each of the 68 maximum correlation values between the left and right Broca's region ROls (136 values total for each subject) were compared between the subjects with pre-determined left-lateralized language and subjects with pre-determined right-lateralized language using unpaired $t$-tests in each cortical region. Of these 68 cortical regions, there were six regions in which there was a significantly higher maximum correlation value $(p<0.05)$ with the left Broca's region ROI in the left-lateralized group as compared with the right-lateralized language group (Fig. 1): left inferior temporal, left lateral orbitofrontal, left pars triangularis, right lateral orbitofrontal, right pars orbitalis and right superior frontal regions. There were no significant differences between groups noted for the maximum correlations with the right Broca's region ROI.

Regarding the multiple comparisons performed in this approach, by chance alone, we would expect for there to be a total of 3.4 cortical regions to have a significant $(p<0.05)$ difference between the two groups (e.g., $0.05 \times 68$ regions). Given that six regions were found, we would surmise that these cortical regions did not show differences between the two groups due to chance alone. As an additional control, the difference in correlation values between the left and right Broca's region ROls and each of the 39 non-cortical Haskins atlas regions (including ventricles and white matter) did not exhibit any significant differences between the two groups.

As a post-hocanalysis, to estimate an approximate predictive ability of the correlations of the above-mentioned six regions with left Broca's region for a subject's language laterality, the average of these six correlation coefficients with left Broca's region was determined for each subject. If the average of these six correlation coefficients was greater than $0.7,{ }^{25}$ then the subject was considered to have left-lateralized language; conversely, if the average of these six correlation coefficients was less than 0.7 , then the subject was considered to have right-lateralized language. By the use of this post-hoc cutoff criteria, 12 of the 15 subjects had concordance between the connectivity of these six regions with left Broca's region. In the three subjects in which this RS connectivity prediction was not concordant with the previous "gold standard" language laterality determination, two of these subjects had tbfMRI performed and one subject had intraoperative language mapping performed to determine language lateralization. It should be noted, however, that this method of the using the RS connectivity values to "predict" a patient's language laterality is linked to the previous analysis, and hence, caution should be taken to not overinterpret these results. ${ }^{26}$

A group-level map was created to compare voxel-wise correlations between the left and right Broca's region ROls and each voxel of the whole brain map between the left- and right-hemispheric language
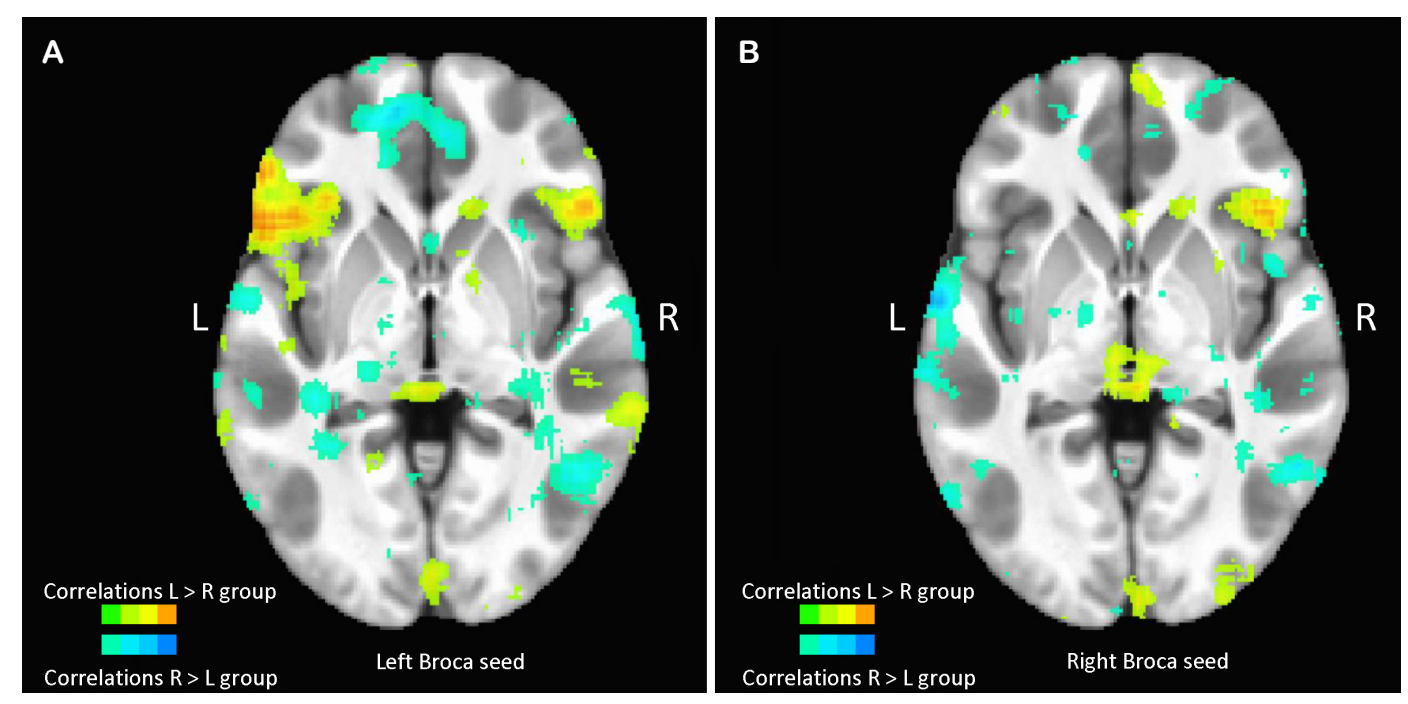

Figure 2. Group analysis: group level maps of differences in voxel-wise correlations between each voxel and the left Broca (A) or right Broca (B) seed regions between the left-lateralized and right-lateralized language groups. These differences in correlations were thresholded at 0.1 . The left hemisphere of each brain is represented on the left. Voxels with a greater correlation value in the left-lateralized language than right-lateralized language group are shown in the upper color bar, with colors ranging from green (smallest correlation differences) to orange (highest correlation differences). Voxels with a greater correlation value in the right-lateralized language than left-lateralized language group are shown in the lower color bar, with colors ranging from light blue (smallest correlation differences) to dark blue (highest correlation differences). Anatomical underlay is the Haskins pediatric anatomical template. 
groups. The unthresholded difference maps are shown in Fig. 2; the colored regions did not survive statistical thresholding, but are shown for visualization purposes. There appear to be trend-level differences in the group maps, with more spatially extensive auto-correlations within the left Broca's region than right Broca's region in the left language group. There is also a higher degree of correlation between the left and right Broca's regions when the left Broca's region is used as a seed in the left language group. Given that these trend-level differences did not survive corrections for multiple corrections, the reader should be cautious to not overinterpret the findings from these group-level difference maps.

To help visualize the potential concordance between the tbfMRI voxel map and the results of the RS left and right Broca's region seed comparison, Fig. 3 shows the tbfMRI and RS maps in one subject. In this subject, LI of this tbfMRI language paradigm was greater than +0.1 , indicating left-lateralized language. In Fig. $3 \mathrm{~A}$, there are voxels within the left inferior frontal cortex and left midline interhemispheric cortex which had a greater response during verb generation than during rest (voxels in purple). In Fig. 3B, the orange voxels represent the areas which had greater correlation with the left Broca's region seed than right Broca's region seed. Similar to the results seen in the above-described group-level map, in this single subject with predetermined left-lateralized language, there appears to be a higher degree of auto-correlation within the left Broca's region (orange voxels) than within the right Broca's region (blue voxels).

\section{Discussion}

We examined differences in language laterality in pediatric epilepsy patients using a novel seed-based RS approach. The current study focused on differences in RS networks in pediatric epilepsy patients due to the critical importance in lateralizing language function in patients who may undergo surgical resection of seizure foci, as well as the difficulty in having children participate in task-based functional studies. A group of nine patients with left-lateralized language were found to have greater voxel-wise correlations than a group of six patients with right-lateralized language between a left hemispheric Broca's region seed and the following six cortical regions: left inferior temporal, left lateral orbitofrontal, left pars triangularis, right lateral orbitofrontal, right pars orbitalis and right superior frontal regions. This finding represents the first evidence known to the authors of differences in seed-based RS networks between pediatric epilepsy patients with left- and right-hemispheric language lateralization

The current study benefits from having a large proportion (40\%) of the studied patients with right-lateralized language function, which is helpful in creating a more balanced view of differences in language
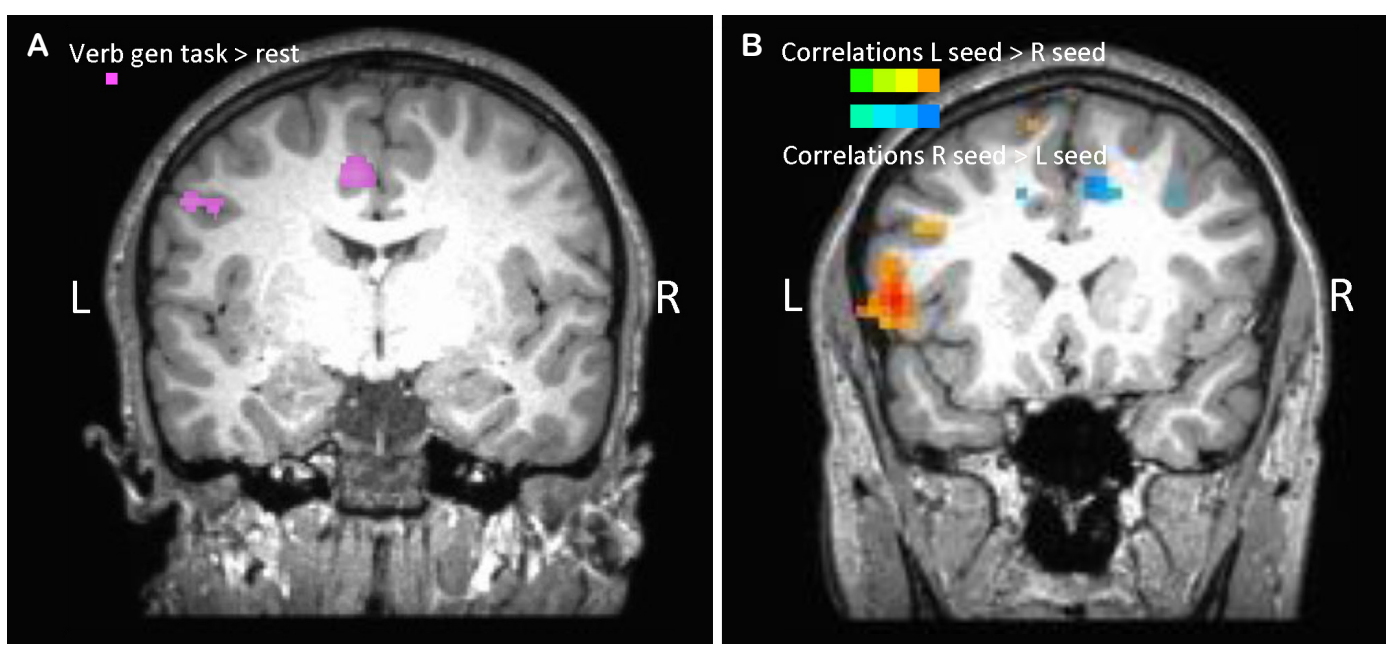

Figure 3. Comparison of task-based fMRI result and resting-state fMRI in a single subject. (A) Map of areas with greater BOLD fMRI activity during verb generation blocks than rest blocks, thresholded at $\mathrm{T}>2$. The laterality index of this tbfMRI language paradigm was greater than +0.1 , indicating left-lateralized language. (B) In the same subject, this map shows the result of subtracting the RS map using the left Broca's region as a seed minus the RS map using the right Broca's region as a seed (left-right maps). The orange regions represent voxels with greater correlation with the left Broca's seed than right Broca's seed, and the blue regions represent voxels with greater correlation with the right Broca's seed than left Broca's seed. The left hemisphere of each brain is represented on the left. A coronal view slice is shown for both the tbfMRI and RS images. fMRI, functional magnetic resonance imaging; BOLD, blood oxygen level-dependent; tbfMRI, task-based functional magnetic resonance imaging; RS, resting state. 
networks. Additionally, our "gold standard" techniques for determining language lateralization included a variety of techniques, including transient lesioning with Wada testing and CSM, which provides evidence for the necessity of cortical areas for language function. The individualized approach to creation of seed ROls using each individual's anatomy allows for the study of language networks which may have significant individual variability. ${ }^{27}$

\section{Interpretation of regional differences}

We found six regions with significantly different connectivity between individuals with left- and right-lateralized language function. This is a modest result, which did not translate to significant voxel-wise comparisons, and some number of these significant regional findings may be due to chance. However, many of the regions we found are consistent with previous literature and our understanding of how these networks should behave in left- and right-lateralization. This may suggest that with efforts to increase signal-to-noise in the measurements (for example, longer scanning runs, and less patient motion), this approach would yield a clinically feasible tool for language lateralization. Interpretations of the significant regions are described further.

In the group of patients with previously-determined left-lateralized language function, the significant correlations in RS activity between the left Broca's region and left pars triangularis likely represents an autocorrelation between the left Broca's region and the individual voxels within this region, given that the left pars triangularis lies within the anatomical Broca's region as defined in this study. This auto-correlation within the left Broca's region is a helpful finding in terms of quality control, as we would expect that the averaged resting state timecourse with left Broca's region should correlate highly with individual voxels within the same region. Another useful quality control measure is the difference between the correlation maps when using left and right Broca's regions seeds, which is consistent with a dataset which is not driven entirely by artifacts and/or noise.

Interestingly, when the right Broca's region was used as a seed, we did not see stronger correlations between the right Broca's region and homologous left Broca's region in the left-lateralized language group; this may be indicative of the right Broca's region not being as central to the language network as the left Broca's region in the left-hemispheric language group. There also appears to be a greater spatial extent of auto-correlation within the right Broca's region seed in the left-lateralized language group, possibly due to the right Broca's region being a less significant hub in the language networks of the right-lateralized language group.

There is evidence of stronger interhemispheric connectivity in the left-lateralized language group as compared with the right-lateralized language group. There was a significant correlation in the left-lateralized language patient group between the left Broca's region and right pars orbitalis of the frontal lobe, which is adjacent to the right Broca's region anatomical region. This correlation between left Broca's region and voxels near the homologous region in the right hemisphere has been found previously. ${ }^{28}$ Additionally, the strong correlation between left Broca's region and the superior frontal cortex in the contralateral hemisphere suggests stronger interhemispheric connectivity within the left-lateralized language group, which is supported by trend-level correlations greater correlations between the left and right Broca's regions in the left-lateralized group noted in our voxel-wise group maps shown in Fig. 2 .

Additionally, the significant correlations seen between left Broca's region and the superior frontal region have been previously reported in a large study of 970 healthy adult subjects, ${ }^{29}$ although it is unknown how many of the previously-studied adult subjects had left-lateralized language. Based on our finding of strong correlations seen between left Broca's region and right superior frontal cortex in the left-lateralized language subjects, we might expect that this finding is concordant with a large cohort of adult subjects with presumably a high proportion of left-lateralized language, as has been found previously with adult language lateralization. ${ }^{30}$

Strong correlations between left Broca's region and the inferior temporal cortex and orbitofrontal regions suggest interactivity between expressive language networks and other networks involved in language interpretation. There were significant correlations between left Broca's region and the left inferior temporal cortex in the patients with left-lateralized language. The left inferior temporal cortex has been found to be involved in reading tasks in adults, ${ }^{31}$ and the strong connectivity between left Broca's region and left inferior temporal cortex in the patients with left-lateralized language in this study suggests a concordance in laterality between expressive speech and reading functions. The strong correlations between left Broca's region and the left and right lateral orbitofrontal regions in the patients with left-lateralized language suggest an interplay between expressive language networks and bilateral orbitofrontal cortical activity in the evaluation of emotional prosody of speech. ${ }^{32}$

There were no cortical regions found which had significant correlations with either the left or right Broca's region within the right-lateralized language group. This finding is supportive of right-lateralized 
language networks being more distributed than the language networks of subjects with left-lateralized language function. ${ }^{33}$

\section{Limitations and additional considerations}

Our findings of differences in RS functional connectivity between epilepsy patients with left- and right-lateralized language could potentially be confounded by the lateralization of seizure onset zones in these patients. Previous study of 50 patients with focal epilepsy showed evidence of language laterality in epilepsy patients being driven by lateralization of a patient's seizure focus, with patients with left-sided seizure foci having a higher likelihood of right-lateralized language, and all patients with a right-sided seizure foci having left-lateralized language. ${ }^{34}$ In this study, however, nearly all patients with left-lateralized language had seizure foci within the left hemisphere, suggesting that language lateralization and differences in RS correlations in this study were not driven solely by the location of seizure foci and epileptogenic networks. Nevertheless, it would be helpful to follow up these findings with a comparison of RS networks between epilepsy patients and healthy controls with right-lateralized language, in order to determine if there are differences in the language networks which may be accounted for by the presence of epileptogenic foci.

These limitations of RS connectivity for the localization of language are significant, though, this method is still worth continued work and exploration given the limitations of the current "gold standard" methods of tbfMRI, CSM, and Wada testing. Many pediatric patients with epilepsy are unable to sit motionless for a long enough period to participate in tbfMRI, limiting the population which may take advantage of this method. With CSM in patients who have intracranial EEG electrodes implanted, there is a risk of inducing seizures with the electrode stimulation; hence, this method is used with caution. Wada testing comes with significant risks to the patient, including stroke and arterial vessel dissection, and cross-flow of the amobarbital to the contralateral hemisphere may render the behavioral results uninterpretable. ${ }^{35}$

This pilot study presents evidence for differences in seed-based RS networks between pediatric epilepsy patients with left- and right-lateralized language function. Our hope will be for further investigations to help establish RS as a method to help in language localization in these patients; while there are currently differences in RS networks noted at a group level, the current technique is not yet applicable at the individual level. One challenge arose in defining the exact locations of the left and right Broca's region seeds, given that there was variability in functional activity across voxels within the anatomically-defined pars triangularis and opercularis. Further research with the RS networks of healthy children could feasibly automate a method to determining a consistent Broca's region seed region across pediatric subjects. There were also issues with motion-related artifacts resulting in datasets which were unusable due to noise. Future work may be able to mitigate the influence of motion with various strategies, including collecting longer scans; training patients prior to the scanning session with mock scanners; or implementing real-time motion tracking software, such as FIRMM, ${ }^{36}$ which would allow operators to identify poor quality scans and repeat them at the time of the MRI session.

However, despite these challenges, the current study shows as a proof of concept in this small cohort that there may be promise to using RS to localize language in pediatric epilepsy patients. The future clinical implications of a task-free method of language localization are multifold; the analysis of RS connectivity as a proxy for language lateralization would provide useful information for epilepsy pre-surgical planning, as well as aid in the planning of tumor resections near potential eloquent cortex in children unable to participate in traditional task-based language localization methods. The RS connectivity method may also be helpful in the future to track longitudinal changes in language localization across time in single subjects following cortical injury or surgical resections. While this technique is still in its infancy, the future uses of a task-free, connectivity-based functional neuroimaging method to localize language are wide-ranging and worthy of investigation.

\section{Acknowledgements}

This research was supported by a Thrasher Research Fund Early Career Award Program grant.

\section{References}

1. Zack MM, Kobau R. National and state estimates of the numbers of adults and children with active epilepsy - United States, 2015. MMWR Morb Mortal Wkly Rep 2017;66:821-5.

2. Berg AT, Langfitt JT, Testa FM, et al. Global cognitive function in children with epilepsy: a community-based study. Epilepsia 2008;49:608-14.

3. Kirsch $M$, Guldenmund $P$, Ali Bahri $M$, et al. Sedation of patients with disorders of consciousness during neuroimaging: effects on resting state functional brain connectivity. Anesth Analg 2017;124:588-98.

4. Ansari AH, Oghabian MA, Hossein-Zadeh GA. Assessment of functional 
and structural connectivity between motor cortex and thalamus using fMRI and DWI. Conf Proc IEEE Eng Med Biol Soc 2011;2011:5056-9.

5. Dosenbach NU, Fair DA, Miezin FM, et al. Distinct brain networks for adaptive and stable task control in humans. Proc Natl Acad Sci U S A 2007; 104:11073-8.

6. Honey CJ, Sporns O, Cammoun L, et al. Predicting human resting-state functional connectivity from structural connectivity. Proc Natl Acad Sci U S A 2009;106:2035-40.

7. Lee TW, Xue SW. Revisiting the functional and structural connectivity of large-scale cortical networks. Brain Connect 2018;8:129-38.

8. Tanaka N, Stufflebeam SM. Presurgical mapping of the language network using resting-state functional connectivity. Top Magn Reson Imaging 2016;25:19-24.

9. Doucet GE, Pustina D, Skidmore C, Sharan A, Sperling MR, Tracy Jl. Resting-state functional connectivity predicts the strength of hemispheric lateralization for language processing in temporal lobe epilepsy and normals. Hum Brain Mapp 2015;36:288-303.

10. Smitha KA, Arun KM, Rajesh PG, Thomas B, Kesavadas C. Resting-state seed-based analysis: an alternative to task-based language $\mathrm{fMRI}$ and its laterality index. ANNR Am J Neuroradiol 2017;38:1187-92.

11. Desai VR, Vedantam A, Lam SK, et al. Language lateralization with resting-state and task-based functional MRI in pediatric epilepsy. J Neurosurg Pediatr 2018;23:171-7.

12. Quinones-Hinojosa A, Ojemann SG, Sanai N, Dillon WP, Berger MS Preoperative correlation of intraoperative cortical mapping with magnetic resonance imaging landmarks to predict localization of the Broca area. J Neurosurg 2003;99:311-8.

13. Pallud J, Zanello M, Kuchcinski G, et al. Individual variability of the human cerebral cortex identified using intraoperative mapping. World Neurosurg 2018;109:e313-7.

14. Akman $\mathrm{Cl}$, Micic $\mathrm{V}$, Quach $\mathrm{M}$, et al. Application of envelope trend to analyze early EEG changes in the frontal regions during intracarotid amobarbital procedure in children. Epilepsy Behav 2015;43:66-73.

15. Cox RW. AFNl: software for analysis and visualization of functional magnetic resonance neuroimages. Comput Biomed Res 1996;29:162-73.

16. Dale AM, Fischl B, Sereno Ml. Cortical surface-based analysis. I. Segmentation and surface reconstruction. Neuroimage 1999;9:179-94.

17. Fischl B, Sereno MI, Dale AM. Cortical surface-based analysis. II: inflation, flattening, and a surface-based coordinate system. Neuroimage 1999:9:195-207.

18. Argall $B D$, Saad $Z$ S, Beauchamp MS. Simplified intersubject averaging on the cortical surface using SUMA. Hum Brain Mapp 2006;27:14-27.

19. Dronkers NF, Plaisant O, Iba-Zizen MT, Cabanis EA. Paul Broca's historic cases: high resolution MR imaging of the brains of Leborgne and Lelong. Brain 2007;130(Pt 5):1432-41.

20. Mclntosh AR, Gonzalez-Lima F. Network interactions among limbic corti- ces, basal forebrain, and cerebellum differentiate a tone conditioned as a Pavlovian excitor or inhibitor: fluorodeoxyglucose mapping and covariance structural modeling. J Neurophysio/ 1994;72:1717-33.

21. Nath AR, Beauchamp MS. Dynamic changes in superior temporal sulcus connectivity during perception of noisy audiovisual speech. J Neurosci 2011;31:1704-14.

22. Mills KL, Goddings AL, Herting MM, et al. Structural brain development between childhood and adulthood: convergence across four longitudinal samples. Neuroimage 2016;141:273-81.

23. Beauchamp MS, Beurlot MR, Fava E, et al. The developmental trajectory of brain-scalp distance from birth through childhood: implications for functional neuroimaging. PLoS One 2011;6:e24981.

24. Molfese PJ, Glen D, Mesite L, Pugh KR, Cox RW. The Haskins pediatric brain atlas. Poster session presented at: 21st Annual Meeting of the Organization for Human Brain Mapping; 2015 Jun; Honolulu, USA.

25. Mukaka MM. Statistics corner: a guide to appropriate use of correlation coefficient in medical research. Malawi Med J 2012;24:69-71.

26. Kriegeskorte N, Simmons WK, Bellgowan PS, Baker Cl. Circular analysis in systems neuroscience: the dangers of double dipping. Nat Neurosci 2009;12:535-40.

27. Seghier ML, Lazeyras F, Pegna AJ, et al. Variability of fMRI activation during a phonological and semantic language task in healthy subjects. Hum Brain Mapp 2004;23:140-55.

28. Bernal $B$, Ardila A, Rosselli M. Broca's area network in language function: a pooling-data connectivity study. Front Psychol 2015;6:687.

29. Tomasi D, Volkow ND. Resting functional connectivity of language networks: characterization and reproducibility. Mol Psychiatry 2012;17:841-54.

30. Knecht $S$, Dräger $B$, Deppe $M$, et al. Handedness and hemispheric language dominance in healthy humans. Brain 2000;123 Pt 12:2512-8.

31. Dong $Y$, Fukuyama $H$, Honda $M$, et al. Essential role of the right superior parietal cortex in Japanese kana mirror reading: an fMRI study. Brain 2000;123(Pt 4):790-9.

32. Wildgruber $D$, Ackermann $H$, Kreifelts B, Ethofer T. Cerebral processing of linguistic and emotional prosody: fMRI studies. Prog Brain Res 2006:156:249-68.

33. Pujol J, Conesa G, Deus J, López-Obarrio L, Isamat F, Capdevila A. Clinical application of functional magnetic resonance imaging in presurgical identification of the central sulcus. J Neurosurg 1998;88:863-9.

34. Berl MM, Balsamo LM, Xu B, et al. Seizure focus affects regional language networks assessed by fMRI. Neurology 2005;65:1604-11.

35. Kundu B, Rolston JD, Grandhi R. Mapping language dominance through the lens of the Wada test. Neurosurg Focus 2019;47:E5.

36. Dosenbach NUF, Koller JM, Earl EA, et al. Real-time motion analytics during brain MRI improve data quality and reduce costs. Neuroimage 2017;161:80-93. 